University of Nebraska - Lincoln

DigitalCommons@University of Nebraska - Lincoln

John R. Hardy Papers

Research Papers in Physics and Astronomy

4-15-1968

Lattice Dynamics and Second-Order Raman Spectrum of CsF

John R. Hardy

University of Nebraska - Lincoln

Arnold Karo

University of California, Livermore, California

Follow this and additional works at: https://digitalcommons.unl.edu/physicshardy

Part of the Physics Commons

Hardy, John R. and Karo, Arnold, "Lattice Dynamics and Second-Order Raman Spectrum of CsF" (1968). John R. Hardy Papers. 12.

https://digitalcommons.unl.edu/physicshardy/12

This Article is brought to you for free and open access by the Research Papers in Physics and Astronomy at DigitalCommons@University of Nebraska - Lincoln. It has been accepted for inclusion in John R. Hardy Papers by an authorized administrator of DigitalCommons@University of Nebraska - Lincoln. 


\title{
Lattice Dynamics and Second-Order Raman Spectrum of CsF $\dagger$
}

\author{
John R. Hardy* and Arnold M. Karo \\ Lawrence Radiation Laboratory, University of California, Livermore, California
}

(Received 16 October 1967)

\begin{abstract}
Results are presented for a precise calculation, based on the deformation dipole model with short-range repulsive interactions between second-neighbor negative ions, of the normal-mode frequency distribution of CsF. The extreme properties of this crystal are reflected in the unusual appearance of the distribution. From these results, we can also predict the form of the second-order Raman spectrum of CsF for two alternative assumptions regarding the Raman polarizability tensor. Both spectra are unusual in that they consist almost entirely of very sharp, clearly separated lines. Furthermore, the spectra corresponding to these two assumptions are very different and should be easily distinguishable experimentally.
\end{abstract}

SOME time ago we presented the results of a system$S$ atic investigation into the lattice dynamics of most of the NaCl-structure alkali halides. ${ }^{1}$ However, certain salts were omitted from this initial study, mainly because of the absence of the required input data, and among these was $\mathrm{CsF}$. This was unfortunate since this compound is, in many respects, an extreme case, with the positive ion being both very much heavier and considerably more polarizable than the negative ion. Since that time, sufficient experimental data ${ }^{2}$ have become available for us to be able to treat this crystal, and we wish to report in this paper the results of our calcula tions. Also we wish to report the results of an associated calculation of the second-order Raman spectrum of this crystal. We believe that these calculations will be of direct and immediate interest to experimental workers studying phonon-assisted electronic transitions at defect

† Work performed under the auspices of the U. S. Atomic Energy Commission.

* Permanent address: Behlen Laboratory of Physics, University of Nebraska, Lincoln, Neb.

1 A. M. Karo and J. R. Hardy, Phys. Rev. 129, 2024 (1963).

${ }^{2}$ C. M. Randall, R. M. Fuller, and D. J. Montgomery, Solid State Commun. 2, 273 (1964). centers in $\mathrm{CsF}$, defect vibrations in $\mathrm{CsF}$, and the secondorder Raman spectrum of CsF. In the first place, apart from a much earlier calculation by one of us (A. M. K.), ${ }^{3}$ there is no other theoretical work on the lattice dynamics of CsF. In the second place, our present work has revealed that the extreme properties of $\mathrm{CsF}$ are reflected both in the calculated frequency spectrum and the calculated second-order Raman spectrum. Both of these have a very striking appearance which should be well worth investigation by any, or all, of the experimental techniques mentioned above.

The calculations presented here were carried out within the framework of the deformation dipole (DD) model which is fully described in an earlier paper. ${ }^{4}$ However, we have recently made an investigation into the Debye-Waller factors of all the cesium halides, ${ }^{5}$ including $\mathrm{CsF}$, and we have found it necessary, particularly for $\mathrm{CsF}$, to include short-range interactions between second-neighbor negative ions to obtain good agreement with experiment (i.e., within the quoted

${ }^{3}$ A. M. Karo, J. Chem. Phys. 33, 7 (1960).

${ }^{4}$ J. R. Hardy, Phil. Mag. 7, 315 (1962).

${ }_{5}^{5}$ A. M. Karo and J. R. Hardy (to be published). 
TABLE I. Input data for $\mathrm{CsF}\left(300^{\circ} \mathrm{K}\right)$ normal-mode calculations.

\begin{tabular}{|c|c|}
\hline $\begin{array}{l}\text { Compressibility }{ }^{\mathbf{a}} \\
\text { Shear modulus } \\
\text { Lattice constant }^{\mathbf{b}} \\
\text { Born-Mayer screening radius } \\
\text { Static dielectric constant } \\
\text { High-frequency dielectric constant } \\
\text { Infrared dispersion frequency } \\
\text { Szigeti effective charge }^{\mathrm{f}} \\
\text { Positive ion polarizabilityd } \\
\text { Negative ion polarizability }\end{array}$ & $\begin{aligned} \beta & =4.000 \times 10^{-12} \mathrm{~cm}^{2} / \mathrm{dyn} \\
C_{44} & =7.750 \times 10^{10} \mathrm{dyn} / \mathrm{cm}^{2} \\
r_{0} & =3.004 \times 10^{-8} \mathrm{~cm} \\
\rho & =0.2709 \times 10^{-8} \mathrm{~cm} \\
\epsilon_{0} & =7.90 \\
\epsilon_{\infty} & =2.158 \\
\omega_{0} & =2.392 \times 10^{13} \mathrm{rad} / \mathrm{sec} \\
s=e^{*} / e & =0.94 \\
\alpha_{+} & =3.137 \times 10^{-24} \mathrm{~cm}^{3} \\
\alpha_{-} & =0.759 \times 10^{-24} \mathrm{~cm}^{3}\end{aligned}$ \\
\hline
\end{tabular}

a Elastic-constant data extrapolated from alkali-fluoride sequence. b R. W. G. Wyckoff, Crystal Structures (Interscience Publishers, Inc.,

New York, 1963), 2nd ed., Vol. I, Table III, 1.

d J. R. Tessman, A. H. Kahn, and W. Shockley, Phys. Rev. 92, 890 (1953).

- Reference 2.

1 M. Born and K. Huang, Dynamical Theory of Crystal Structures (Oxford University Press, New York, 1954), Eq. (9.31).

experimental error). We have therefore included these in the present calculations.

The two disposable force constants involved are fitted to the shear modulus $C_{44}$ and the infrared dispersion

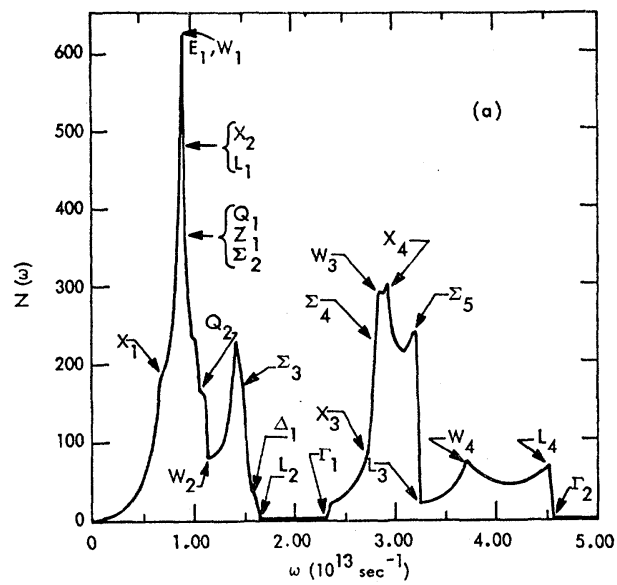

(b)

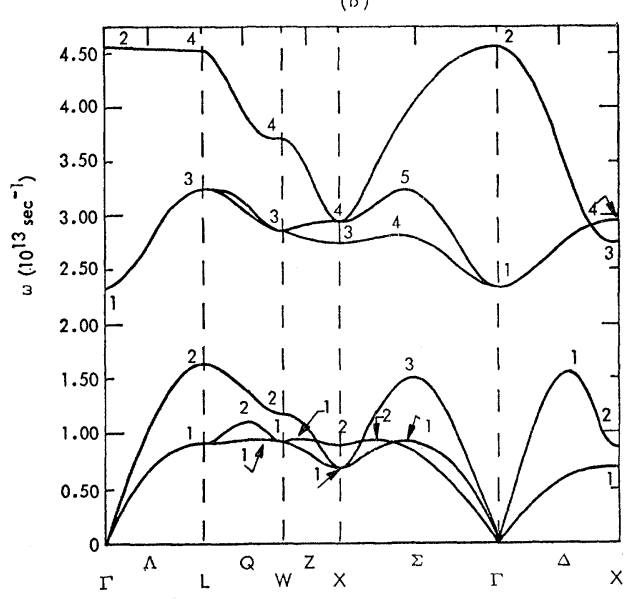

Fig. 1. (a) Gaussian-smoothed $300^{\circ} \mathrm{K}$ frequency spectrum of $\mathrm{CsF}$ showing the principal critical points (c.p.) labeled to correspond to those in the phonon-dispersion curves of (b). (b) $300^{\circ} \mathrm{K}$ phonon-dispersion curves for $\mathrm{CsF}$ with c.p. indicated; these correspond to the c.p. marked in (a) (e.g., the c.p. labeled 1 at the symmetry point $X$ corresponds to the $X_{1}$ c.p. in the spectrum while the c.p. labeled 1 along the $\Delta$ axis corresponds to the $\Delta_{1}$ c.p. on the frequency spectrum).
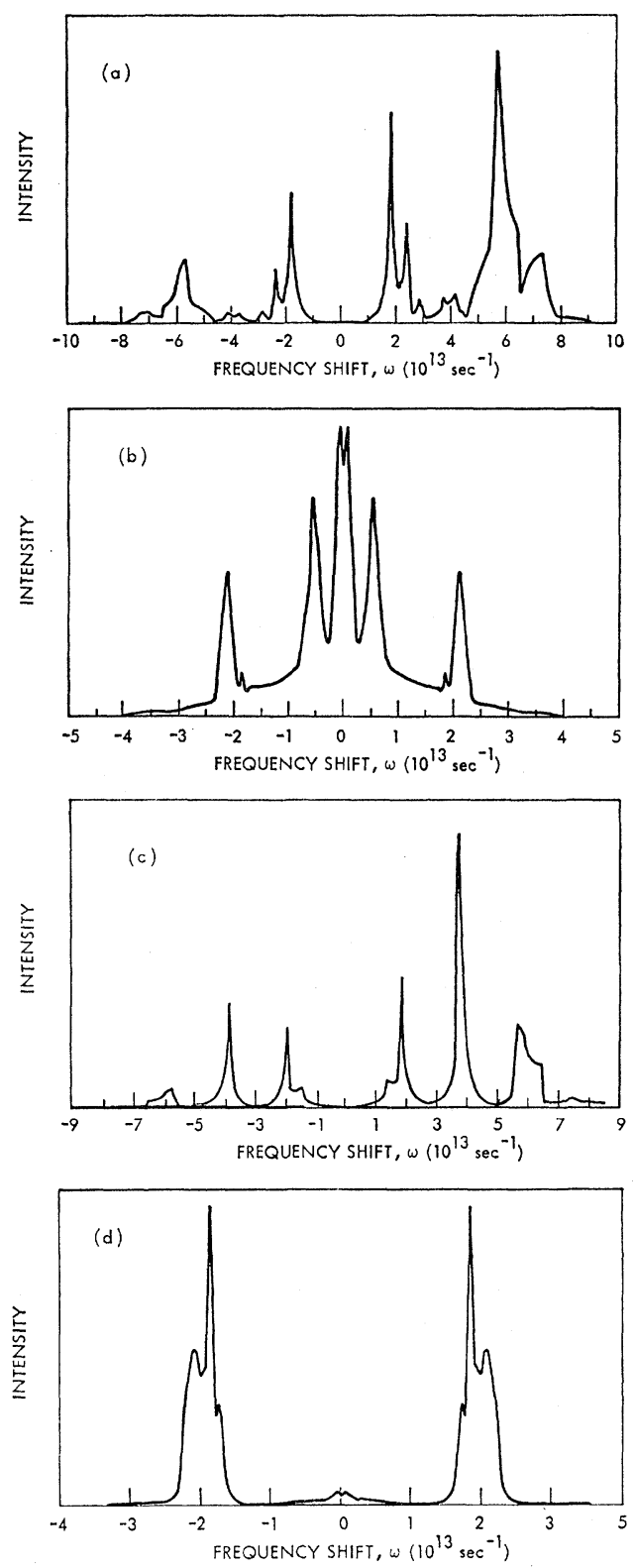

FIG. 2. (a) Predicted $300^{\circ} \mathrm{K}$ Stokes and anti-Stokes components of the second-order Raman spectrum of $\mathrm{CsF}$ on the basis of the first polarizability tensor model: variation 1 . (b) Predicted $300^{\circ} \mathrm{K}$ difference bands in the second-order Raman spectrum of $\mathrm{CsF}$, again on the basis of variation 1 . (c) The same as (a) above, but predicted on the basis of variation 2 . (d) The same as (b) above, but predicted from variation 2 . (Note that in all four cases the intensity scale is arbitrary, but linear, and differs on all four figures, being chosen in each case for convenience in plotting. Intensity differences between the stokes and anti-stokes components of the difference bands are not shown.)

frequency $\omega_{0}$. (We have assumed that all the interionic potentials are central, and that our model therefore automatically satisfies the Cauchy relation $C_{12}=C_{44}$, a reasonable approximation for CsF.) The input parameters required for this modification of the DD model are shown in Table I. The calculations were carried out for 
a uniform sample of 64000 wave vectors $\mathbf{q}$ in the first Brillouin zone and the six normal mode eigenfrequencies determined for each point. Then, in order to obtain a smooth distribution function, instead of the conventional histogram method we represented each sample frequency by a Gaussian entered on the exact frequency. The resultant distribution, shown in Fig. 1, is a superposition of all these Gaussians with their common halfwidth chosen to produce the maximum smoothing consistent with the preservation of genuine structure. Dispersion curves were also calculated along the principal symmetry directions, and the results are shown in Fig. 1. The principal Van Hove singularities, ${ }^{6}$ associated with symmetry points and axes, are labeled in accordance with the notation of Bouckaert et al., ${ }^{7}$ subscripts indicating the order of increasing frequency. As can be seen, the spectrum has several extreme features, e.g., the two very sharp acoustic-mode peaks, the large gap between optic and acoustic branches, and the striking, almost rectangular, peak in the optic branch. These features, if genuine, should certainly manifest themselves in both the phonon-assisted optical transition spectra of defects in $\mathrm{CsF}$ and in impurity-induced infrared lattice absorption. ${ }^{8}$

Recently we have also completed a calculation of the second-order Raman spectrum of $\mathrm{NaF},{ }^{9}$ and have obtained very good agreement with experiment. We therefore carried out a similar calculation for CsF, the results of which are shown in Fig. 2. It is assumed that the temperature of measurement is $300^{\circ} \mathrm{K}$ and that we are dealing with radiation incident along a $\langle 100\rangle$ crystal axis and scattered through $90^{\circ}$ along a $\langle 010\rangle$ axis. Under these circumstances, our assumptions regarding the Raman polarizability tensor predict that the scattered radiation will be completely polarized and therefore the calculated curves refer only to this component. Two separate choices of the polarizability tensor elements, which we believe represent two opposite extremes in the

${ }^{6}$ L. Van Hove, Phys. Rev. 89, 1189 (1953).

${ }^{7}$ L. P. Bouckaert, R. Smoluchowski, and E. Wigner, Phys. Rev. 50, 58 (1936).

${ }^{8}$ W. E. Bron and M. Wagner, Phys. Rev. 139, A233 (1965).

${ }^{9}$ J. R. Hardy, A. M. Karo, I. W. Morrison, C. T. Sennett, and J. P. Russell (to be published). behavior of these quantities, were taken, and the predicted spectra for both variations (1 and 2) are shown in Fig. 2. (For the actual construction of the polarizability tensors, see Ref. 9.)

Again we have used a Gaussian smoothing technique, and for clarity we have shown the difference spectra (i.e., that part arising from processes in which one phonon is created and another destroyed) separately from the combination spectra, which involve either double-phonon creation or destruction. Both types of spectra have a very unusual appearance, since instead of having relatively smooth structure they consist of discrete, very sharp lines. In addition, unlike the results for $\mathrm{NaF}$ where the combination spectra for variations 1 and 2 were very similar, the present data show very different combination as well as difference spectra. Because of this, it seems to us that it would be very worthwhile to have an experimental test of our predicted spectra using a modern laser Raman spectrograph. If the spectrum is indeed strongly polarized for the $\langle 100\rangle$ scattering geometry, then it should easily be possible to discriminate between the two theoretical variations. It should be stressed, however, that our results are contingent on the validity of our polarizability tensor model which assumes only first-neighbor interactions. ${ }^{9}$ This assumption, while apparently valid for $\mathrm{NaF}$, may well be less trustworthy for CsF, where the dominant polarizability is that of the positive ion. However, we would emphasize that reliable data on the relative influence of first- and more-distant-neighbor displacements on the Raman polarizability of a given ion in a given crystal, as one proceeds through a sequence of crystals, are of primary importance in understanding the second-order Raman effect. The only way such information can be obtained is by predicting the Raman spectra on the basis of a series of assumptions and testing prediction against experiment. Thus, our primary object in presenting these calculations of the Raman spectrum of $\mathrm{CsF}$ is to demonstrate the logical consequences of making the same assumptions about the nature of the crystal Raman polarizability tensor for $\mathrm{CsF}$ at the heavy end of the fluoride sequence as for $\mathrm{NaF}$ at the light end. 